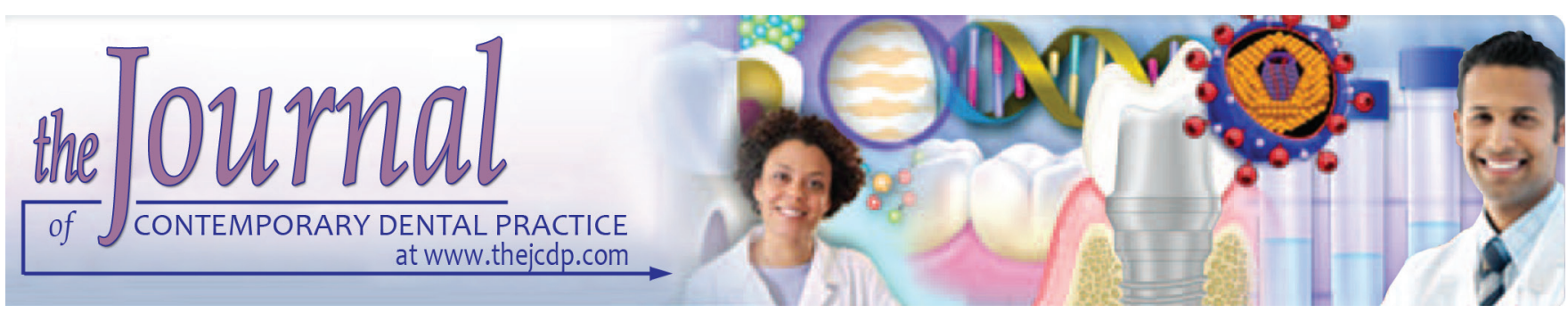

\title{
A Solitary Phlebolith in the Buccal Mucosa: Report of a Rare Entity and Clinicopathologic Correlation
}

\author{
${ }^{1} \mathrm{~A}$ Nagaraja, ${ }^{2} \mathrm{~N}$ Govindraj Kumar, ${ }^{3} \mathrm{~B}$ Jambukeshwar Kumar, ${ }^{4}$ Raghavendra M Naik, ${ }^{5} \mathrm{Y}$ Jyoti Sangineedi
}

\begin{abstract}
Pathological conditions can give rise to calcifications within oral mucosa representing either a local or systemic disturbance. Inflammation, trauma, debris acting as nidus and vascular lesions have been attributed as principal causes for occurrence of calcifications within the oral mucosa. Occurrence of multiple calcified thrombi (phleboliths) is considered pathognomonic for hemangiomas and vascular malformations in the oral and maxillofacial region. Isolated occurrence of phlebolith in oral mucosa though very rare, especially without any underlying vascular lesions, can be diagnostically challenging. Either a traumatic association at that site or a hemangioma of childhood that has regressed once the individual became an adult are the possible explanations suggested for the occurrence of these unique solitary phleboliths. Histologically, an "onion-ring"-like concentric lamellar fibrosis around a central core with varying amounts of calcifications and presence of minute vascular channels within or around calcified lamellae is characteristic for phlebolith. There is a high propensity for misdiagnosing solitary phlebolith located in sites like the buccal mucosa where various other pathologic soft-tissue calcifications, such as sialoliths, calcified lymph nodes, traumatic myositis ossificans, etc. can occur and they too appear radiopaque in radiographs. Besides, the absence of any associated underlying vascular lesion adds to the misperception. In such cases, histopathological examination with routine hematoxylin and eosin staining alone may not be sufficient to determine the accurate diagnosis. Allied clinical history and immunohistochemistry can aid to arrive at the final diagnosis. We report such a case of nonvascular lesion-associated solitary
\end{abstract}

\footnotetext{
1,2,5 Department of Oral and Maxillofacial Pathology, Vishnu Dental College and Hospital, Bhimavaram, Andhra Pradesh India

${ }^{3}$ Department of Oral Surgery, Vishnu Dental College and Hospital, Bhimavaram, Andhra Pradesh, India

${ }^{4}$ Department of Oral Medicine and Radiology, Vishnu Dental College and Hospital, Bhimavaram, Andhra Pradesh, India

Corresponding Author: A Nagaraja, Professor, Department of Oral and Maxillofacial Pathology, Vishnu Dental College and Hospital, Bhimavaram, Andhra Pradesh, India, e-mail: nagsdoc@gmail.com
}

phlebolith in the right buccal mucosa of a healthy 49-year-old male patient and discuss its differential diagnosis with emphasis on histological presentation.

Keywords: Histopathology, Isolated phlebolith, Pathologic oral soft-tissue calcifications, Vascular lesions.

How to cite this article: Nagaraja A, Kumar NG, Kumar BJ, Naik RM, Sangineedi YJ. A Solitary Phlebolith in the Buccal Mucosa: Report of a Rare Entity and Clinicopathologic Correlation. J Contemp Dent Pract 2016;17(8):706-710.

Source of support: Nil

Conflict of interest: None

\section{INTRODUCTION}

Pathologic calcified structures within the oral mucosal soft tissues are indeed rare entities. They could represent a local nonspecific response or be associated with significant underlying pathology. Patient approach and treatment vary greatly depending on the cause of softtissue calcifications. ${ }^{1}$ The soft tissues of the cheek rarely contain lesions that include calcifications. Among them, phleboliths associated with vascular lesions are the most frequent. ${ }^{2}$ However, it requires differentiation from other calcifications that occur in the same area, such as sialoliths, dystrophic/metastatic calcifications, traumatic myositis ossificans, calcified lymph nodes, healed acne lesions, cysticercosis, and pilomatrixoma of the skin. ${ }^{3}$ Although plain radiographs, ultrasonography, and other advanced imaging techniques like computed tomography (CT) with contrast, magnetic resonance imaging (MRI) are useful in differentiating these lesions, histological evaluation aided by immunohistochemistry can clinch the diagnosis. ${ }^{4}$

Multiple phleboliths associated with vascular malformations and hemangiomas of the oral cavity are well documented. Reports on isolated occurrence of phleboliths are very few. Herein, we report a case of an isolated phlebolith in the buccal mucosa not associated with any vascular lesion and discuss its clinicopathologic correlations. 


\section{CASE REPORT}

A49-year-old male patient reported to our institution with a complaint of an asymptomatic nodular mass in the right cheek region incidentally noted a year back, which was initially small and increased to the present size. No history of trauma/pain could be elicited. The medical history was not contributory. Intraorally, on inspection, a normal appearing mucosal surface was seen. But, on palpation, a well-defined, oval, firm, non-tender movable nodule, in the right buccal mucosa posterior to the last molars, about $2 \times 2 \mathrm{~cm}$ in size was identified. Soft-tissue calcification was considered as the provisional diagnosis. Complete hemogram and serum calcium levels were well within normal values. Through an intraoral surgical approach, the lesion was excised in toto (Fig. 1). The specimen received was measuring $2.5 \times 1.5 \times 0.8 \mathrm{~cm}$ and was oval, firm to hard in consistency (Fig. 2). Upon grossing the cut surfaces were smooth and revealed a glistening creamish white-colored

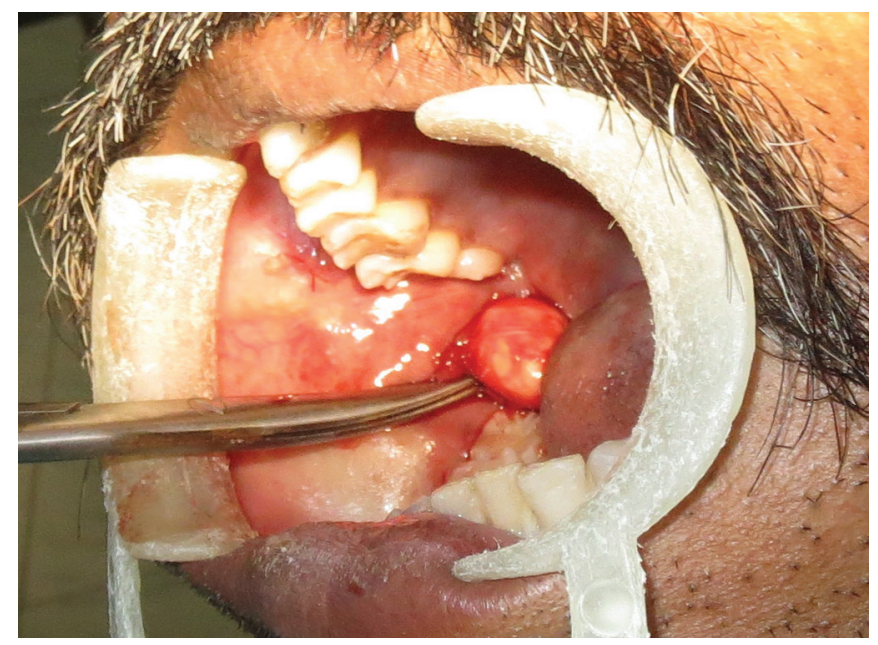

Fig. 1: Clinical photograph showing the submucosal mass during surgical excision

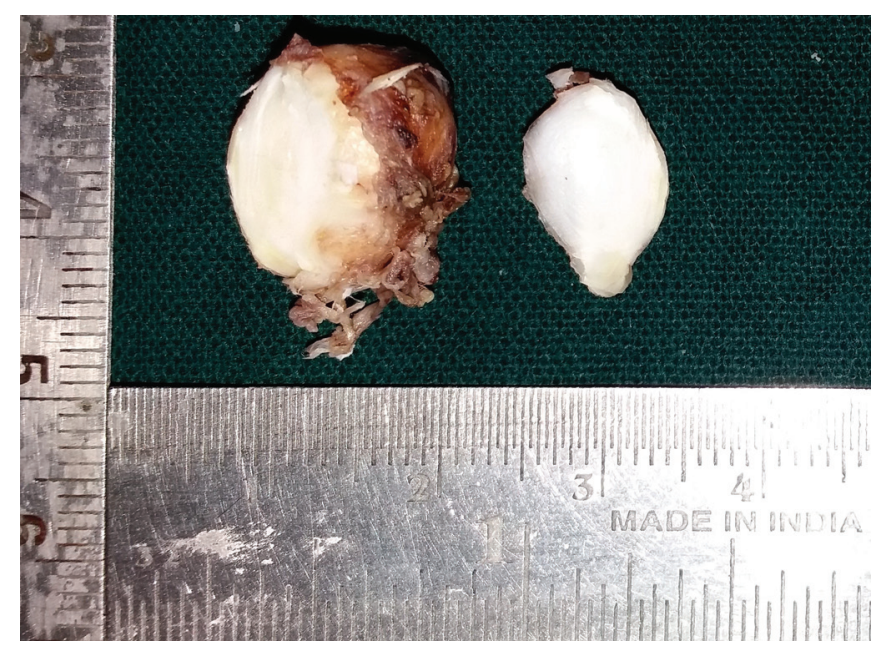

Fig. 3: The cut surfaces of the gross specimen revealing glistening white appearance calcification (Fig. 3). Specimen radiograph showed a radiopaque center with surrounding "onion-ring"-like concentric radiolucent and radiopaque laminations (Fig. 4). Hematoxylin and eosin (H\&E)-stained sections revealed an organizing thrombus with dense basophilic calcifications at the center surrounded by lamellated concentric eosinophilic hyalinized intima (Fig. 5). Peripherally and in some areas among the laminations, extravasated RBCs and thin-walled endothelial cell lined capillaries were observed, and some of them engorged with blood (Fig. 6). Focal immunolocalization of CD34 in blood vessels within lamellated structures confirmed the diagnosis of a phlebolith (Fig. 7). Postoperative follow-up for a year showed no recurrence.

\section{DISCUSSION}

Phleboliths are pathological, calcified vascular thrombi formed due to stasis of blood. Hemangiomas and

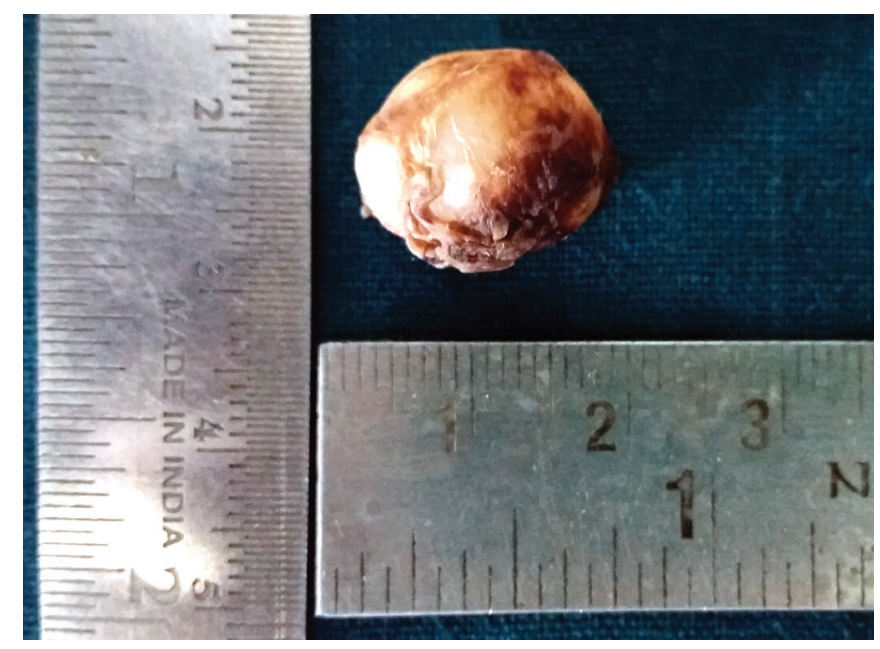

Fig. 2: Brownish white oval gross specimen

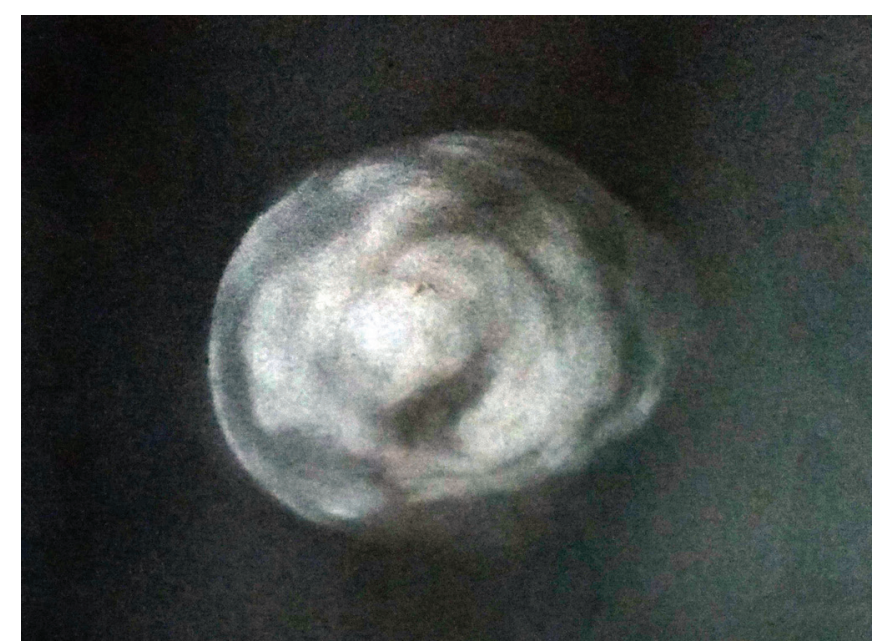

Fig. 4: Specimen radiograph showing central dense opaque area surrounded by laminar calcifications with a smooth peripheral margin 


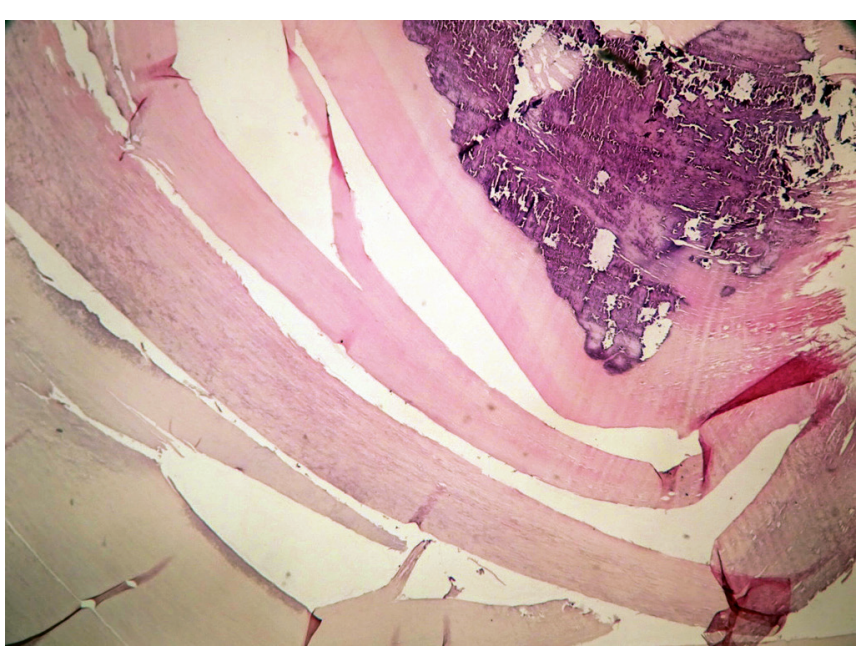

Fig. 5: Microscopic appearance of the phlebolith showing central dense calcification and onion ring pattern of lamellar arrangement $\mathrm{H} \& \mathrm{E}, 20 \times$

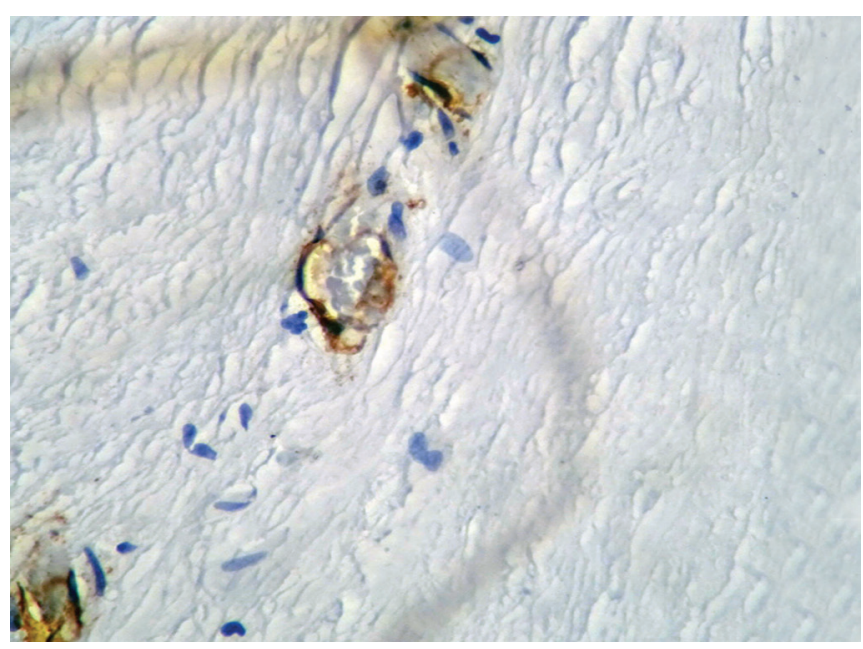

Fig. 7: Immunohistochemistry showing positivity for vascular endothelium stained with anti-CD34 40x

developmental vascular malformations of the head and neck region are well documented to reveal the presence of multiple phleboliths within them. Trauma and regression of childhood hemangiomas of the oral mucosa are considered causative for occurrence of isolated phlebolith. ${ }^{2-6}$ $\mathrm{X}$-ray diffraction and cone beam tomography analysis on phlebolith morphology have demonstrated presence of calcium fluorohydroxyapatite as the main constituent, and the larger the size of the phlebolith, greater is the laminated structural form and radiopacity. 5,7

Phleboliths commonly occur in vascular malformations of splenic and pelvic veins where they were initially identified by Canstatt in 1843 while in the maxillofacial location by Kirmission in $1905 .{ }^{8}$ Phleboliths are innocuous during formation and only on attaining a certain size, do they elicit radiological and clinical features. They appear as small round radiopaque nodules with varying degree of densities, the configuration of which reflects their growth pattern and differs from that of a sialolith, which

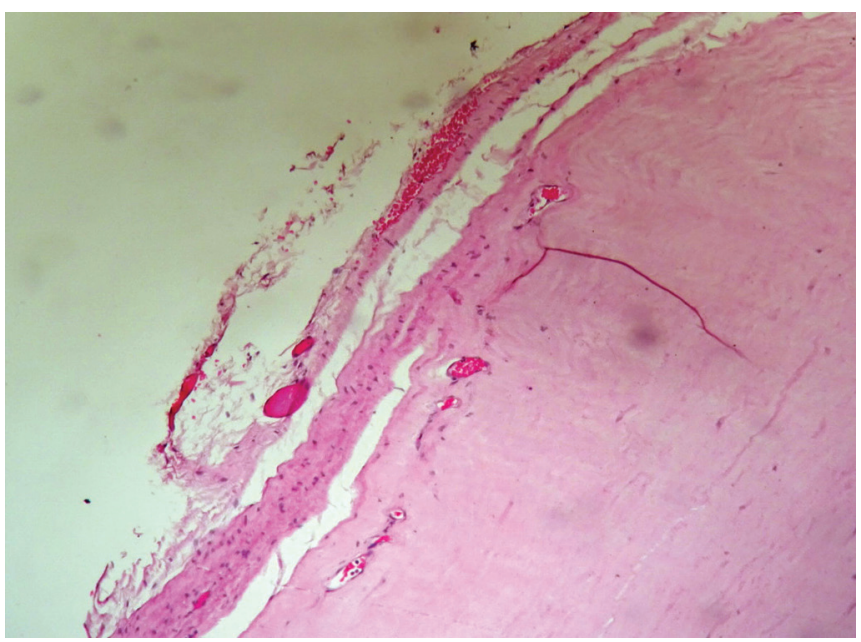

Fig. 6: Microscopic appearance at the periphery of the phlebolith showing minute engorged blood vessel within and outside the concentric lamellae H\&E, 20x

is uniformly radiopaque and elongated. The radiographic appearance of concentric laminations of radiopaque and radiolucent areas correspond to the alternating high and low mineral content laminations that characterize the histologic appearance (Onion ring like) of a phlebolith. $6,7,9$

The pathogenesis of phlebolith was first espoused by Ribbert in the year 1917. A vascular stasis with a resultant sluggish blood flow is pivotal for the formation of a thrombus. The stasis can result because of tortuous blood vessels within a hemangioma or sluggishness of blood flow within vascular malformations. Hence, many phleboliths are found associated with hemangiomas and vascular malformations, especially in the head and neck region. The thrombus that forms undergoes a slow calcification process with calcium carbonate and calcium sulfates deposited within, forming the core of the phlebolith. This process of calcification continues and extends to the periphery with concomitant lamellar fibrosis giving rise to histological onion ring like appearance. Repetition of this phenomenon brings about an enlargement of the phlebolith. ${ }^{2,3,6,10}$ Lima et al reviewed English literature for oral phleboliths from 1970 to 2013 and found 29 cases of phleboliths, out of which five cases showed multiple and three cases presented with solitary phlebolith which were not associated with any vascular malformations. ${ }^{9}$ In our case the solitary phlebolith was found in a 49 -year-old male patient and was not associated with any vascular malformation or history of trauma. Therefore, the occurrence of phlebolith as a reminiscent of resolved erstwhile childhood hemangiomas can be discounted in our case. ${ }^{7}$

Nevertheless, the specimen should be differentiated from other pathologic calcifications that occur in the oral mucosa. Sialoliths are apatite structures with their mineral composition constituting of calcium carbonate and calcium sulfate similar to that of phleboliths. Although 
they are frequently seen in intra- or extra-glandular ductal system of major salivary glands, occasionally they arise in minor salivary glands of oral cavity, including those of the buccal mucosa. Clinical symptoms of ductal obstruction, sialography, unique "onion-ring"-like radiographic appearance of phlebolith can aid to differentiate the two lesions. In our case, the lesion was located in the buccal mucosa away from the vicinity of the Stenson's duct and symptoms of ductal obstruction were absent. Acellular dense annular circumferential rings of calcification around an amorphous central core along with the presence of ductal structures associated with varying degrees of inflammation are microscopic features of a sialolith ${ }^{11}$ in contrast to a phlebolith which reveals an organizing thrombus with lamellar calcifications and densely fibrosed intima with or without secondary calcifications. Immunostaining utilizing anti-CD34/CD31 antibodies reveals presence of vascular tissue within the phlebolith, especially those not associated with vascular lesions, thereby distinguishing the two lesions. Other authors have also employed immunohistochemistry with smooth muscle marker (SMA) and histochemical Wiegert's and Masson's Trichrome staining to confirm the diagnosis of phlebolith. $^{2,7}$

Calcified cutaneous acnes are more superficial occurring on the skin in clinical presentation and are usually multiple in numbers and thus can easily be excluded from the diagnosis. ${ }^{2}$ Pilomatrixoma or calcifying cutaneous tumor of Malherbe are benign neoplasms of the hair follicle at times revealing calcifications, presenting as hard nodular movable swelling in the subcutaneous part of the skin external to buccal mucosa. Presence of basaloid proliferation, ghost cells, and ossifying areas within the tumor tissue is pathognomonic for pilomatrixoma while no such histological presentation is observed in phleboliths. $^{12}$

Heterotropic ossification popularly called as myositis ossificans is a trauma associated bone or cartilage, producing reactive lesion uncommon in the head and neck region. Masseter and other facial muscles of younger individuals are involved occasionally. Linear striated appearance or feathery kind of calcification along the muscle fibers are initial radiological manifestation. Older lesions reveal single large radiopaque mass often with central radiolucency. Clinically, it presents as minimally movable firm mass which can be palpated beneath the skin or mucosa. Histological evidence of woven or lamellated bone or cartilage in a background of proliferative fibroblasts are conspicuous for traumatic myositis ossificans while lack of bone-like calcifications is pertinent to phleboliths. Moreover, our case presented with radiopaque central core and the patient was 49 years old with no history of trauma. ${ }^{13}$
Calcifications within lymph nodes are a rare phenomenon in the head and neck area and are usually consequent to postinflammatory lymphadenopathy and secondary heterotrophic calcification. They commonly involve cervical lymph nodes, with metastatic deposits from distant malignancies, spread of squamous cell carcinomas of the head and neck, granulomatous lesions including tuberculosis, postirradiation treatment sequelae, and Hodgkin's lymphomas are some of the attributed etiologies. Moreover, calcified lymph nodes are yellowish, larger in size compared to phlebolith and are readily identifiable by radiography while histological evaluation can relate to the cause of such calcifications, especially when metastasis is concerned. In our case, the patient was healthy with no significant medical history and histological evidence of inflammation was also lacking. ${ }^{14,15}$

Metastatic and dystrophic calcifications too can lead to formation of calcified structures within the oral mucosal tissue. Dystrophic calcifications occur due do deposition of calcium salts within necrotic and degenerating tissue while metastatic calcifications occur due to increased calcium levels in the blood. Cases of dystrophic calcifications involving masseter muscle have been reported in younger individuals. Histologically, they reveal irregular multiple calcifications within a dense fibrous tissue. Our case revealed a single foci of calcification with lamellated structures around the calcification. ${ }^{16}$ Metastatic calcifications are reported in lungs, beneath the skin, kidneys, and other tissues but presenting clinically as an oral lesion is extremely rare. Chronic renal failure, milk-alkali syndrome, extensive bone malignancy, hypervitaminosis $\mathrm{D}$ are some of the conditions known to cause metastatic calcifications. However, they are multiple or single big calcified masses and microscopically reveal foreign-body giant cells around irregular extensive amorphous calcifications in a dense fibrous connective tissue background associated with variable chronic inflammatory infiltrate, which can be readily distinguished from characteristic onion-ring-like appearance of phleboliths. ${ }^{17}$ Our case was bereft of any necrotic areas in the specimen and patient's blood investigation picture was noncontributory toward increased levels of calcium.

Cysticercosis, a parasitic infection occurring in endemic areas where man is the intermediate host for transmission of adult Taenia Solium (Pork Tapeworm), is rare in the oral mucosa. Interestingly, submucosal nodular swelling with no surface alterations of the oral mucosa is the most common mode of clinical presentation. Tongue is most commonly affected site while infrequent cases are reported in buccal mucosa also. History and histological examination will render a conclusive diagnosis with the aid of imaging and serological investigations. A capsule 
of fibrous connective tissue around a cystic cavity containing dead Cysticercus cellulosae (larva) which undergoes eventual secondary calcification, focal collection of eosinophils among a dense inflammatory infiltrate are some of the salient histological features of cysticercosis which help in easy differentiation from a phlebolith. ${ }^{18}$

Occasionally, phleboliths are a feature of some syndromes like Maffucci syndrome, the Klippel-TrenaunayWeber syndrome, the Sturge-Weber syndrome, and the rubber-bleb syndrome. However, they usually occur in multiple numbers in these syndromes, unlike in our case which presented as a single solitary lesion. ${ }^{6,19}$

Phleboliths are not known to recur after surgical enucleation, but their presence usually denotes an attendant vascular lesion, since many cases of phleboliths have been reported in oral cavity in conjunction with hemangiomas and vascular malformations. ${ }^{4,5,9}$ Our case is interesting due to the fact that no vascular abnormality was observed and extremely few cases of isolated phleboliths of the oral cavity are reported in the scientific literature. Buccal mucosa is inadvertently exposed to masticatory trauma leading to damage of blood vessels with subsequent submucosal extravasation, stasis, and eventual calcification of thrombus formed inside the tissue compartment. This process might be the etiopathogenesis of our case. ${ }^{3}$

\section{CONCLUSION}

Conventional histopathology with the aid of immunostaining along with pertinent clinical information can sufficiently narrow down the diagnosis of oral soft-tissue calcifications. Even though rare, isolated phlebolith not associated with vascular lesions must be considered among the differentials for calcified masses in the oral cavity, particularly when present in locations subjected to trauma. Immunohistochemistry employing vascular tissue markers is of value in diagnosing such phlebolith not associated with vascular lesions.

\section{REFERENCES}

1. Hussmann J, Russell RC, Kucan JO, Khardori R, Steinau HU. Soft-tissue calcifications: differential diagnosis and therapeutic approaches. Ann Plast Surg 1995 Feb;34(2):138-147.

2. Kato H, Ota Y, Sasaki M, Arai T, Sekido Y, Tsukinoki K. A phlebolith in the anterior portion of the masseter muscle. Tokai J Exp Clin Med 2012 Apr 20;37(1):25-29.

3. Mandel L, Perrino MA. Phleboliths and the vascular maxillofacial lesion. J Oral Maxillofac Surg 2010 Aug;68(8):1973-1976.
4. Zengin AZ, Celenk P, Sumer AP. Intramuscular hemangioma presenting with multiple phleboliths: a case report. Oral Surg Oral Med Oral Pathol Oral Radiol 2013 Jan;115(1):e32-e36.

5. Gooi Z, Mydlarz WK, Tunkel DE, Eisele DW. Submandibular venous malformation phleboliths mimicking sialolithiasis in children. Laryngoscope 2014 Dec;124(12):2826-2828.

6. Zachariades N, Rallis G, Papademetriou J, Konsolaki E, Markaki S, Mezitis M. Phleboliths. A report of three unusual cases. Br J Oral Maxillofac Surg 1991 Apr;29(2):117-119.

7. Gouvêa Lima Gde M, Moraes RM, Cavalcante AS, Carvalho YR, Anbinder AL. An Isolated phlebolith on the lip: an unusual case and review of the literature. Case Rep Pathol 2015;2015: 507840, 5 .

8. Scolozzi P, Laurent F, Lombardi T, Richter M. Intraoral venous malformation presenting with multiple phleboliths. Oral Surg Oral Med Oral Pathol Oral Radiol Endod 2003 Aug;96(2):197-200.

9. Mohan RP, Dhillon M, Gill N. Intraoral venous malformation with phleboliths. Saudi Dent J 2011 Jul;23(3):161-163.

10. Saha A, Mohapatra M, Patra S, Saha K. Phlebolith in arteriovenous malformation in buccal fat pad masquerading sialolith: a rare case report. Contemp Clin Dent 2015 Apr-Jun;6(2): 254-256.

11. Lee LT, Wong YK. Pathogenesis and diverse histologic findings of sialolithiasis in minor salivary glands. J Oral Maxillofac Surg 2010 Feb;68(2):465-470.

12. Jaiswal AA, Garg AK, Membally R, Anand T, Mohanty MK. Ossifying pilomatrixoma with marrow formation of the left cheek region - case report with review of literature. Egypt J Ear Nose Throat Allied Sci 2015;16(2):193-199.

13. Gnepp DR. Diagnostic surgical pathology of head and neck. 1st ed. Philadelphia (PA): WB Saunders; 2001. p. 161-162.

14. Fujimura K, Nishida M, Son A, Kunishima F, Segami N, Lizuka T, Kitaichi M. Heterotopic calcification in advanced cervical lymph nodes with metastasis from squamous cell carcinoma of the tongue: report of two cases. Oral Oncol Extra 2004 Nov;40(10):117-122.

15. Eisenkraft BL, Som PM. The spectrum of benign and malignant etiologies of cervical node calcification. AJR Am J Roentgenol 1999 May;172(5):1433-1437.

16. Mohiuddin SA, Badal S, Doiphode A, Sultana S. Multiple supramassetric dystrophic calcinosis. Ann Maxillofac Surg 2012 Jan-Jun;2(1):74-76.

17. Verma DK, Thelekkat Y, Bansal S. Metastatic calcification of floor of the mouth secondary to chronic renal failure - report of a rare case with atypical presentation. Dentomaxillofac Radiol 2015 May;44(5):20140023.

18. Elias FM, Martins MT, Foronda R, Jorge WA, de Araújo NS. Oral cysticercosis: case report and review of the literature. Rev Inst Med Trop Sao Paulo 2005 Mar-Apr;47(2):95-98.

19. Nozaki T, Nosaka S, Miyazaki O, Makidono A, Yamamoto A, Niwa T, Tsutsumi Y, Aida N, Masaki H, Saida Y. Syndromes associated with vascular tumors and malformations: a pictorial review. Radiographics 2013 Jan-Feb;33(1):175-195. 